\title{
REGULARITY CONDITION BY MEAN OSCILLATION TO \\ A WEAK SOLUTION OF THE 2-DIMENSIONAL HARMONIC HEAT FLOW INTO SPHERE
}

\author{
Masashi Misawa \\ Department of Mathematics, School of Science, \\ Kumamoto University \\ Kumamoto 860-8555, Japan \\ Takayoshi Ogawa \\ Mathematical Institute, \\ Tohoku University \\ Sendai 980-8578, Japan
}

\begin{abstract}
We show a regularity criterion to the harmonic heat flow from 2-dimensional Riemannian manifold $M$ to a sphere. It is shown that a weak solution of the harmonic heat flow from 2-dimensional manifold onto a sphere is regular under the criterion

$$
\int_{0}^{T}\|\nabla u(\tau)\|_{B M O_{r}}^{2} d \tau<\infty
$$

where $B M O_{r}$ is the class of functions of bounded mean oscillations on $M$. A sharp version of the Sobolev inequality of the Brezis-Gallouet type is introduced on $M$. A monotonicity formula by the mean oscillation is established and applied for proving such regularity criterion for weak solutions as above.
\end{abstract}

\section{INTRODUCTION}

In this paper, we consider the regularity problem of weak solutions to the time dependent harmonic heat flow from a two dimensional Riemannian manifold to a sphere: Let $(M, g)$ denotes a Riemannian manifold. The harmonic heat flow from $M$ to $\mathbb{S}^{m}$ is originally considered as the $L^{2}$-gradient flow of the Dirichlet energy:

$$
\frac{1}{2} \int_{M} \nabla_{k} u_{i}(x) \nabla_{l} u_{j}(x) g_{k, l}(x) \sqrt{g(x)} d x,
$$

where $\nabla_{k} u_{i} \nabla_{l} u_{j} g_{k, l}=\nabla_{x_{k}} u_{j} \nabla_{x_{l}} u_{j} g_{k, l}$ denotes the quadratic form associated with the base manifold $(M, g)$ and $g(x)=\left|\operatorname{det}\left[g_{k, l}(x)\right]\right|$. The critical points of this functional are subject to the harmonic map from $(M, g)$ to $\mathbb{S}^{m}$,

$$
\left\{\begin{aligned}
-\Delta_{M} u=u(\nabla u, \nabla u)_{g}, \quad x \in M, & \\
u: M \rightarrow \mathbb{S}^{m} &
\end{aligned}\right.
$$

where $\Delta_{M}=\frac{1}{\sqrt{g}} \nabla_{k}\left(\sqrt{g} g_{k, l} \nabla_{l}\right)$ denotes the Laplace-Beltrami operator on $M$ with $u(\nabla u, \nabla u)_{g}=$ $u_{i} \sum_{1 \leq l, m \leq n, 1 \leq j \leq m}\left(g_{l m} \nabla_{l} u_{j}, \nabla_{m} u_{j}\right)$ denotes the second fundamental form of the sphere in $\mathbb{R}^{m+1}$.

Key words: the harmonic heat flow, regularity condition, the bounded mean oscillation. AMS Subject Classification: primary 35K55, 58E20 secondary 58J35, 46E30. 
The resulting evolution equation from the $L^{2}$-gradient flow of the above energy functional is the harmonic heat flow as follows:

$$
\left\{\begin{array}{c}
\partial_{t} u-\Delta_{M} u=u(\nabla u, \nabla u)_{g}, \quad t>0, x \in M, \\
u(t, x): \mathbb{R}_{+} \times M \rightarrow \mathbb{S}^{m}, \quad t>0, x \in M, \\
u(0, x)=u_{0}(x), \quad x \in M .
\end{array}\right.
$$

Eells-Sampson [15] firstly considered this equation for the sake of constructing a homotopy from arbitrary initial data to the stationary harmonic map. By a formal observation, the following type of the energy inequality is immediately obtained:

$$
\|\nabla u(t)\|_{L^{2}(M)}^{2}+2 \int_{0}^{t}\left\|\partial_{t} u(\tau)\right\|_{L^{2}(M)}^{2} d \tau \leq\left\|\nabla u_{0}\right\|_{L^{2}(M)}^{2} \equiv E_{0}, \quad t \in[0, T] .
$$

Based on the above energy inequality, a weak solution is constructed in the space $u \in L^{\infty}\left(0, T ; \dot{H}^{1}\left(M ; \mathbb{S}^{m}\right)\right)$ with $\partial_{t} u \in L^{2}\left(0, T ; L^{2}\left(M ; \mathbb{S}^{m}\right)\right)$. When the dimension of the base manifold $M$ is 2, then Struwe [37] constructed the weak solution which is piecewise smooth in time variable. On the other hand, the existence of a partially regular global weak solution was established by Chen-Struwe [13] by the penalty method. If the initial data is smooth, a smooth local solution exists by using the Bochner type formula (see for example Eells-Sampson [15] and Struwe [37]). This time-local smooth solution is belonging to $u \in W^{1, \infty}\left(M ; \mathbb{S}^{m}\right)$ and the maximal existence time is characterized by $\left\|\nabla u_{0}\right\|_{\infty}$.

The regularity of the weak solution fails in general because of the existence of a blowing up solution for a large initial data. The example for the map from $B_{1}(0) \subset \mathbb{R}^{n}$ to a sphere was shown by Coron-Ghidaglia [14] for $n \geq 3$ and Chang-Ding-Ye [11] for $n=2$. However, some smallness assumption on the initial data or integrablity condition on the solution itself are capable to give the regularity.

One of the regularity class for the weak solution to (1.2) is the class introduced by Struwe [37]: $V=\left\{u: M \rightarrow \mathbb{S}^{2}: \nabla u \in L^{\infty}\left(0, T ; L^{2}(M)\right), \partial_{t} u, \Delta_{M} u \in L^{2}\left(0, T ; L^{2}(M)\right)\right\}$. Our aim here is to extend this class larger when $\operatorname{dim} M=2$ in terms of the mean oscillation of the solution. In fact in [27], it is proved that a time-local smooth solution $u:\left[0, T_{0}\right) \times \mathbb{R}^{n} \rightarrow \mathbb{S}^{m}$ of (1.2) for some $T_{0}$ can be extended over $\left[T_{0}, T_{0}+T^{\prime}\right)$ for some $T^{\prime}>0$, provided

$$
\int_{0}^{T_{0}}\|\nabla u(t)\|_{B M O}^{2} d t<\infty .
$$

Here BMO is the space of functions of bounded mean oscillation defined by

$$
f \in L_{l o c}^{1}\left(\mathbb{R}^{n}\right), \quad\|f\|_{B M O} \equiv \sup _{x, R} \frac{1}{\left|B_{R}\right|} \int_{B_{R}(x)}\left|f(y)-\bar{f}_{B_{R}(x)}\right| d y<\infty,
$$

where $\bar{f}_{B_{R}}$ is the average of $f$ over $B_{R}(x)=\left\{y \in \mathbb{R}^{n} ;|x-y|<R\right\}$.

The above results can be compared with the existing blow-up solutions for (1.2). CoronGhidaglia [14] and Chen-Ding [10] showed that there exists a finite time blowing up solution to (1.2) for $n \geq 3$. For $n=2$, Chang-Ding-Ye [11] constructed a blowing up solution from a smooth data (see for the regularity of the harmonic maps, Schoen-Uhlenbeck [34], Hélein [22], 
Evans [17] and for the evolution case, Feldman [18]). The solution satisfies

$$
\int_{0}^{T}\|\nabla u(t)\|_{\infty}^{\theta} d t=\infty \quad(\theta>1)
$$

where $T>0$ is the expected blow-up time. We simply remark that for two dimensional case, if we make the stronger regularity assumption;

$$
\int_{0}^{T}\|\Delta u(t)\|_{2}^{2} d t<\infty
$$

then by the embedding

$$
\int_{0}^{T}\|\nabla u(t)\|_{B M O}^{2} d t<\infty
$$

and our criterion gives the regularity. If a weak solution satisfies the following integrablity condition;

$$
\int_{0}^{T}\|\nabla u(t)\|_{4}^{4} d t<\infty
$$

then the condition (1.5) is fulfilled and the solution has to be smooth near $t=T$. This is nothing but the criterion from the scaling invariant norm

$$
\int_{0}^{T}\|\nabla u(t)\|_{p}^{\theta} d t<\infty, \quad \frac{2}{\theta}+\frac{n}{p}=1 .
$$

The criterion (1.4) is a weaker assumption and outside of this circle of the regularity criterion.

Analogous situation can be observed in the theory of a weak solution to the incompressible fluid mechanics. For the viscous incompressible fluid governed by the Navier-Stokes equation;

$$
\left\{\begin{array}{l}
\partial_{t} u-\Delta u+u \cdot \nabla u+\nabla p=0, \quad t>0, x \in \mathbb{R}^{n}, \\
\quad \operatorname{div} u=0, \quad t>0, x \in \mathbb{R}^{n}, \\
u(0, x)=u_{0}(x),
\end{array}\right.
$$

it is well known that there exists a global weak solution $u$ based on an analogous energy inequality to (1.2) due to Leray [25];

$$
\|u(t)\|_{2}^{2}+2 \int_{0}^{t}\|\nabla u(\tau)\|_{2}^{2} d \tau \leq\left\|u_{0}\right\|_{2}^{2}
$$

Although a full regularity of the weak solution to (1.6) still remains open, there are some sufficient conditions for the regularity of the solution in terms of a semi-norm invariant under the scaling that maintain the equations. For the Navier-Stokes case, the equation is invariant under the scaling; $u_{\lambda}(t, x)=\lambda u\left(\lambda^{2} t, \lambda x\right), p_{\lambda}(t, x)=\lambda^{2} p\left(\lambda^{2} t, \lambda x\right)(\lambda>0)$. Hence a criterion by the space-time norms such as

$$
\int_{0}^{T}\left\||\nabla|^{\alpha} u(t)\right\|_{p}^{\theta} d t<\infty, \quad \frac{2}{\theta}+\frac{n}{p}=1+\alpha, \quad 2 \leq \theta<\infty,
$$

gives the regularity of a weak solution. This is known as the Serrin condition (Prodi [30], Ohyama [26], Serrin [35], Giga [20] ). By observing the analogous scaling $u \rightarrow u_{\lambda}=u\left(\lambda^{2} t, \lambda x\right)$ that preserves the equation (1.2), it is expected that there is a regularity criterion for (1.2) under the condition;

$$
\nabla u \in L^{\theta}\left(0, T ; L^{p}\left(\mathbb{R}^{n}\right)\right), \quad \frac{2}{\theta}+\frac{n}{p}=1, \quad n<p \leq \infty .
$$


Those conditions are corresponding to the Serrin criterion and enough to show the regularity of the strong solution to (1.2).

In view of the limiting condition to (1.6) the Leray-Hopf weak solution to (1.6) is regular under the corresponding regularity assumption for vorticity:

$$
\int_{0}^{\infty}\|\operatorname{rot} u(\tau)\|_{B M O} d \tau<\infty .
$$

Hence it is expected that under the analogous regularity condition such as (1.4), certain weak solutions to (1.2) are shown to be regular. This is shown in [27] as an extension result for the smooth (strong) solution for (1.2). However to show (1.4) being the criterion for a weak solution to (1.2) is not so straightforward, indeed. For the case of the Navier-Stokes equation, the proof is heavily depending on the fact that any weak solution corresponds the smooth solution for certain time interval. This partial uniqueness result fails in general for a weak solution to (1.2) even in the two dimensional domain, by the nonuniqueness result due to Bertsch, Dal Passo and van der Hout [4] (for the another interesting nonuniqueness, see Topping [40], also reder to Freire [19] and Bertisch-Dal Passo-Pisante [5]).

However, we may show certain kind of weak solutions to (1.2) are regular under the same assumption (1.4) when we restrict the base manifold as in 2 dimensions. To state this precisely, we introduce the definition of the weak solution:

Definition. A map $u: M \rightarrow \mathbb{S}^{m}$ is a weak solution of $(1.2)$ over $[0, T)$ if

(1) $\nabla u \in L^{\infty}\left(0, T ; L^{2}(M)\right)$ and $\partial_{t} u \in L^{2}\left(0, T ; L^{2}(M)\right)$.

(2) $\|\nabla u(t)\|_{L^{2}(M)}^{2} \leq\left\|\nabla u_{0}\right\|_{L^{2}(M)}^{2} \equiv E_{0}$ holds for all $t \geq 0$.

(3) $u$ satisfies the harmonic heat flow in the sense of distribution:

For all $\phi \in C_{0}^{1}\left([0, T) ; C_{0}^{\infty}(M)^{n}\right)$,

$-\int_{0}^{T} u(\tau) \cdot \partial_{t} \phi(\tau) d x d \tau+\int_{0}^{T}(\nabla u(\tau), \nabla \phi(\tau))_{g} d \tau=\int_{0}^{T} u(\nabla u, \nabla u)_{g} \phi(\tau) d x d \tau+u_{0} \cdot \phi$

where $(\cdot, \cdot)_{g}$ is the $L^{2}$ inner product on $M$.

The existence of a weak solution satisfying the above first two conditions are proved in most general case by Chen-Struwe [13]. The strong solution that has finite point singularity has been discussed by Struwe [37] (also see Schoen-Uhlenbeck [34]).

To state our regularity criterion, we introduce the bounded mean oscillation on the Riemannian manifold $M$. According to Brezis-Nirenberg [7], we define the following class associated with $M$.

Definition. Let $u$ be a map from $M$ to sphere $\mathbb{S}^{m}$. For $r<1$, a map $u$ is in a (locally) bounded mean oscillation over $M ; B M O_{r}=B M O_{r}\left(M ; \mathbb{S}^{m}\right)$ if

$$
\|u\|_{B M O_{r}(M)} \equiv \sup _{x \in M, R<r} \frac{1}{\left|B_{R}(x)\right|} \int_{B_{R}(x)}\left|u(y)-\bar{u}_{B_{R}}\right| \sqrt{g(y)} d y<\infty,
$$

where $B_{R}(x)$ is a geodesic ball on $M$ with radius $R>0$ and

$$
\bar{u}_{B_{R}}=\frac{1}{\left|B_{R}\right|} \int_{\substack{B_{R}(x) \\ 4}} u(y) \sqrt{g(y)} d y
$$


with $\left|B_{R}\right|$ is the geodestic volume of the ball. The conventional class $B M O$ denotes the functions over $\mathbb{R}^{n}$ that is defined by taking the suprimum in the above definition over all $x \in M$. In what follows, we concentrate the case when the base manifold $M$ is either 2 dimensional sphere $\mathbb{S}^{2}$ or the flat torus $\mathbb{T}^{2}$. The result is also true for the Euclidian space $\mathbb{R}^{2}$ or a 2-dimensional compact Riemannian manifold. However to avoid the complicated presentation, we describe all the expression in the following section only for the case $M=\mathbb{T}^{2}$. We suppose an extra regularity condition to be satisfied for the weak solution, which is associated with the scaling invariant norm involving $B M O$.

Theorem 1.1 (Limiting regularity criterion). Let $M$ be either $\mathbb{S}^{2}, \mathbb{T}^{2}$ or $\mathbb{R}^{2}$ and $u$ be a weak solution to (1.2) defined in the above. If, for some $T>0$, the solution u satisfies

$$
\int_{0}^{T}\|\nabla u(\tau)\|_{B M O_{r}(M)}^{2} d \tau<\infty
$$

then the solution is regular up to $t=T$. Namely, $u \in C\left((0, T] ; W^{1, \infty}\left(M ; \mathbb{S}^{m}\right)\right) \cap C^{1}\left((0, T] ; W^{2, \infty}\left(M ; \mathbb{S}^{m}\right)\right)$. In the other words, if the solution blows up at some time $t \leq T$, then

$$
\int_{0}^{T}\|\nabla u(\tau)\|_{B M O_{r}(M)}^{2} d \tau=\infty .
$$

In particular, if for any $t>0$ and some $T>0$

$$
\int_{t}^{t+T}\|\nabla u(\tau)\|_{B M O_{r}\left(\mathbb{S}^{2}\right)}^{2} d \tau<\infty
$$

then the weak solution is globally regular.

The basic idea to show the regularity is twofold. One is to employ a critical type of the Sobolev inequalities. Brezis-Gallouet [6] and Brezis-Wainger [8] firstly showed the following inequality: For $s>n / p$,

$$
\|f\|_{\infty} \leq C\left(1+\left\||\nabla|^{n / p} f\right\|_{p}\left(\log \left(e+\|f\|_{W^{s, p}}\right)\right)^{1-1 / p}\right)
$$

for $f \in W^{s, p}\left(\mathbb{R}^{n}\right)$. Analogous but vector version of this inequality was found by Beale-KatoMajda [2]: For $f \in W^{s, p}\left(\mathbb{R}^{n} ; \mathbb{R}^{n}\right)$ with $\operatorname{div} f=0$,

$$
\|\nabla f\|_{\infty} \leq C\left(1+\|\nabla f\|_{2}+\|\operatorname{rot} f\|_{\infty} \log \left(e+\|f\|_{W^{s, p}}\right)\right)
$$

and used for the regularity theory of the fluid mechanics. Kozono-Taniuchi [24] generalized the above inequality involving BMO; for $s>n / p+1, f \in W^{s, p}$ with $\operatorname{div} f=0$,

$$
\|\nabla f\|_{\infty} \leq C\left(1+\|\operatorname{rot} f\|_{B M O} \log \left(e+\|f\|_{W^{s, p}}\right)\right)
$$

and Kozono-Ogawa-Taniuchi [23] in Besov spaces. We first introduce a generalized version of the critical Sobolev inequality in the Lizorkin-Triebel space (cf. Ogawa [27]) that includes all the above inequalities. It then, turns out that the second exponent of those spaces gives an explicit dependence of the power of the logarithmic term to the higher regularity, which reflects hypotheses on the integral exponent in the time direction of those criteria. In the following section, we show a refined version of the Beale-Kato-Majda and Kozono-Taniuchi type 
inequalities and give some discussion. Then in the successive section, we recall the regularity criterion for the strong (smooth) solution to (1.2).

To extend the above observation into a general weak solution, we need to employ the second ingredient which is a version of the monotonicity formula and so called $\varepsilon$-regularity argument by means of the mean oscillation of the gradient of the solution. Namely there exist some small constants $\varepsilon_{0}>0$ and $R_{0}>0$ such that if for some $R<R_{0}$,

$$
\frac{1}{R^{2}} \int_{t_{0}-R^{2}}^{t_{2}} \int_{B_{R}\left(x_{0}\right)}\left|\nabla u(t, x)-\overline{\nabla u}_{R}\right|^{2} d x d t<\varepsilon_{0}
$$

with $\overline{\nabla u}_{R}$ is roughly speaking the average of $\nabla u$ over $\left(t_{0}-R^{2}, t_{0}\right) \times B_{R}\left(x_{0}\right)$, then the solution is regular around the space time point $\left(t_{0}, x_{0}\right)$. This is an improved version of the existing regularity criterion (see [38]) and itself seems to be interesting, since such regularity condition as above may lead to the improvement of the blow up rate of the solution at a singular point (refer to [41, Theorem 1.6, Remark 1.8, pp.283-284]). The main part is to obtain the monotonicity formula for the mean oscillation of the smooth solution. The $\varepsilon$-regularity criterion is stated together in the section 4 . This $\varepsilon$ - regularity criterion is used to derive that the possible singular time is in fact a finite number and then we may apply the continuing result for the smooth solution before the possible blowing up time. This is presented in the last section.

Before closing this section, we introduce some notations. $B_{R}\left(x_{0}\right)$ is a disk in $\mathbb{R}^{2}$ centered at $x_{0} \in \mathbb{R}^{2}$ with radius $R$ and $B_{R}=B_{R}(0) . I_{R}\left(t_{0}\right)$ is a time interval $I_{R}\left(t_{0}\right)=\left(t_{0}-R^{2}, t_{0}\right)$. We freely use the parabolic neighborhood of $\left(t_{0}, x_{0}\right)$, denoted by $P_{R}\left(t_{0}, x_{0}\right)=I_{R}\left(t_{0}\right) \times B_{R}\left(x_{0}\right)$. $\mathcal{F} f$ and $\hat{f}$ denotes the Fourier transform of $f \cdot\langle x\rangle=\left(1+|x|^{2}\right)^{1 / 2}$. We define a saturated logarithmic function $\log ^{+} t=\log (e+t)$. The usual Sobolev space $W^{s, p}\left(\mathbb{R}^{n}\right)$ is abbreviated as $W^{s, p}$ with the norm

$$
\|f\|_{W^{s, p}} \equiv \| \mathcal{F}^{-1}\left(\langle\xi\rangle^{s} \hat{f}(\cdot) \|_{p}\right.
$$

while $W^{k, p}\left(M ; \mathbb{R}^{m+1}\right)$ is the Sobolev space over $M$ whose norm is

$$
\|f\|_{W^{k, p}} \equiv\left\|\nabla_{g}^{\alpha} f(\cdot)\right\|_{L^{p}(M)}
$$

for $1<p<\infty$ and $\alpha$ as multi-indices with $|\alpha|=k, k \in \mathbb{N} \cup\{0\}$.

We recall the Paley-Littlewood dyadic decomposition (cf. Stein [36], Bergh-Löfström [3]). Let $\phi_{j}(x)$ be the inverse Fourier transform of the $j$-th component of the dyadic decomposition i.e., $\sum_{j=-\infty}^{\infty} \hat{\phi}\left(2^{-j} \xi\right)=1$ except $\xi=0$, where the support of $\hat{\phi}(\xi)$ is located on $2^{-1}<|\xi|<2$. We denote $\psi(x)=\mathcal{F}^{-1}[\hat{\psi}(\xi)](x)$, where

$$
\hat{\psi}= \begin{cases}1, & |\xi|<1 \\ \text { smooth, } & |\xi|<2 \\ 0, & |\xi|>2\end{cases}
$$

Set $\psi_{j}=\mathcal{F}^{-1}\left[\hat{\psi}\left(2^{j} \xi\right)\right](x)$. For a smooth function $f$, we set $\Phi_{j} f=\phi_{j} * f$ and $\Psi f=\psi * f$. The homogeneous Besov space $\dot{B}_{p, \rho}^{s}\left(\mathbb{R}^{n}\right)$ is defined through the full-dyadic decomposition by

$$
\dot{B}_{p, \rho}^{s}\left(\mathbb{R}^{n}\right)=\left\{f \in \mathcal{Z}^{\prime}\left(\mathbb{R}^{n}\right):\|f\|_{\dot{B}_{p, \rho}^{s}}<\infty\right\},
$$


where $\|f\|_{\dot{B}_{p, \rho}^{s}}=\left(\sum_{j=-\infty}^{\infty} 2^{j s \rho}\left\|\phi_{j} * f\right\|_{p}^{\rho}\right)^{1 / \rho}$ and $\mathcal{Z}^{\prime}\left(\mathbb{R}^{n}\right)$ denotes the dual space of $\mathcal{Z}\left(\mathbb{R}^{n}\right)=\{f \in$ $\mathcal{S} ; D^{\alpha} \hat{f}(0)=0 ; \alpha \in \mathbb{N}^{n}$ multi-index $\}$ and can be identified by the coefficient space of $\mathcal{S}^{\prime} / \mathcal{P}$ with the polynomials space $\mathcal{P}$. The homogeneous Lizorkin-Triebel space $\dot{F}_{p, \rho}^{s}\left(\mathbb{R}^{n}\right)$ is similarly defined by

$$
\dot{F}_{p, \rho}^{s}\left(\mathbb{R}^{n}\right)=\left\{f \in \mathcal{Z}^{\prime}\left(\mathbb{R}^{n}\right):\|f\|_{\dot{F}_{p, \rho}^{s}}<\infty\right\},
$$

where $\|f\|_{\dot{F}_{p, \rho}^{s}}=\left\|\left(\sum_{j=-\infty}^{\infty} 2^{j s \rho}\left|\phi_{j} * f\right|^{\rho}\right)^{1 / \rho}\right\|_{p}$ and $1 \leq p<\infty, 1 \leq \rho \leq \infty(1 \leq \rho<\infty$ if $p=\infty)$. We refer to Triebel [42] for more detailed properties of those spaces. We also abbreviate $B M O\left(\mathbb{R}^{n}\right)$ and $B M O_{r}\left(\mathbb{S}^{n}\right)$ as $B M O$ and $B M O_{r}$, respectively.

\section{Sharp Version of Logarithmic Inequality}

In this section, we give a sharp version of the logarithmic Sobolev inequality on sphere. The original type of Sobolev inequality was found by Brezis-Gallouet [6] and Brezis-Wainger [8] (see also Engler [16]). And the similar type of inequality was established Beale-Kato-Majda [2], Kozono-Taniuchi [24] and Kozono-Ogawa-Taniuchi [23]. We first give the sharp version of the inequality shown in [27].

Lemma 2.1 (Sharp version of logarithmic inequality [27]). (1) For any $p, \rho, \sigma \in[1, \infty], q \in$ $[1, \infty), \nu \leq \sigma_{1}, \sigma_{2}, \nu<\rho$ and $\gamma>0$, there exists a constant $C$ which is only depending on $n, p$ such that for $f \in \dot{F}_{p, \sigma_{1}}^{n / p+\gamma}\left(\mathbb{R}^{n}\right) \cap \dot{F}_{p, \sigma_{2}}^{n / p-\gamma}\left(\mathbb{R}^{n}\right)$, we have for $\gamma<\gamma^{\prime}$

$$
\|f\|_{\dot{F}_{\infty, \nu}^{0}} \leq C\|f\|_{\dot{F}_{\infty, \rho}^{0}}\left(1+\left(\frac{1}{\gamma} \log ^{+} \frac{\left\|f_{+}\right\|_{\dot{F}_{p, \sigma_{1}}^{n / p+\gamma^{\prime}}}+\left\|f_{-}\right\|_{\dot{F}_{p, \sigma_{2}}^{n / p-\gamma^{\prime}}}}{\|f\|_{\dot{F}_{\infty, \rho}^{0}}}\right)^{1 / \nu-1 / \rho}\right),
$$

where $f_{+}=\sum_{j \geq 0} \phi_{j} * f$ and $f_{-}=\sum_{j \leq 0} \phi_{j} * f$.

REMARK 1. In the theorem, the assumption $\gamma>0$ is essential. The analogous version of the inequality (2.1) in the Besov space was proved in Ogawa-Taniuchi [29].

The relation between the Lizorkin-Triebel spaces and $B M O\left(\mathbb{R}^{n}\right)$ is well understood. The following result is due to Peetre and Triebel (see also Bui Hui Qui [9]).

Proposition 2.2 (Triebel [42]). $\dot{F}_{\infty, 2}^{0}\left(\mathbb{R}^{n}\right) \simeq B M O\left(\mathbb{R}^{n}\right)$. Namely there exists a constants $C$ such that

$$
C^{-1}\|f\|_{\dot{F}_{\infty, 2}^{0}} \leq\|f\|_{B M O} \leq C\|f\|_{\dot{F}_{\infty, 2}^{0}}
$$

¿From (2.1) and the equivalence between $\dot{F}_{\infty, 2}^{0}\left(\mathbb{R}^{n}\right) \simeq B M O\left(\mathbb{R}^{n}\right)$ and $\dot{F}_{\infty, \infty}^{0}\left(\mathbb{R}^{n}\right) \simeq \dot{B}_{\infty, \infty}^{0}\left(\mathbb{R}^{n}\right)$ it is explicitly shown that the difference between $L^{\infty}\left(\mathbb{R}^{n}\right), B M O\left(\mathbb{R}^{n}\right)$ and the Besov space $\dot{B}_{\infty, \infty}^{0}\left(\mathbb{R}^{n}\right)$ as follows. This is a version of the sharp form of the Kozono-Taniuchi inequality (1.11). 
Proposition 2.3. For $\kappa>0$ and $\hat{f}(0)=0$,

$$
\|f\|_{\infty} \leq C\left(1+\|f\|_{B M O}\left(\frac{1}{\kappa} \log ^{+}\left(\left\|f_{+}\right\|_{\dot{F}_{\infty, 2}^{\kappa}}+\left\|f_{-}\right\|_{\dot{F}_{\infty, 2}^{-\kappa}}\right)\right)^{1 / 2}\right)
$$

Especially, if $\nabla f \in W^{1, q}\left(\mathbb{R}^{n}\right) \cap L^{2}\left(\mathbb{R}^{n}\right)$ for $n<q$, we have

$$
\|\nabla f\|_{\infty} \leq C(q)\left(1+\|\nabla f\|_{B M O}\left(\log ^{+}\left(\|\nabla f\|_{W^{1, q}}+\|f\|_{\infty}\right)\right)^{1 / 2}\right) .
$$

REMARK 2. The condition $\nabla f \in L^{2}$ for the second inequality is redundant but we assume it for the simplicity. The last inequality (2.2) improves the related logarithmic inequality due to Beale-Kato-Majda and Kozono-Taniuchi. Reminding the Brezis-Gallouet inequality,

$$
\|f\|_{\infty} \leq C\left(1+\|f\|_{2}+\|\nabla f\|_{2}\left(\log ^{+}\|\Delta f\|_{2}\right)^{1 / 2}\right), \quad f \in H^{2}\left(\mathbb{R}^{2}\right),
$$

it should be noted that the inequality (2.3) has the same order to the higher regular term despite of the dimension independence, although it is substituted by the Dirichlet norm instead of BMO semi-norm.

Proof of Proposition 2.3. Noting the inequality

$$
x\left(\log \left(e+\frac{y}{x}\right)\right)^{1 / 2} \leq \begin{cases}C\left(1+x(\log (e+y))^{1 / 2}\right), & \text { for } 0<x \leq 1, \\ C x(\log (e+y))^{1 / 2}, & \text { for } 1<x,\end{cases}
$$

and observing that

$$
\|f\|_{\infty}=\left\|\sum_{j=-\infty}^{\infty} \phi_{j} * f\right\|_{\infty} \leq\|f\|_{\dot{F}_{\infty, 1}^{0}}
$$

when $\hat{f}(0)=0$, the first inequality (2.2) is an immediate consequence of (2.1) with $\nu=1$ and $\rho=2$ and Proposition 2.2.

For the second modification (2.3), we show

$$
\lim _{k \rightarrow \infty}\left\|\nabla f-\left(\sum_{j \geq-k} \phi_{j} * \nabla f\right)\right\|_{\infty}=0 .
$$

Introducing a smooth function $\psi(x)$ such that $\hat{\psi}(\xi)=\left\{\begin{array}{ll}1 & |\xi| \leq 1 / 2 \\ 0 & |\xi| \geq 1\end{array}\right.$ and set $\widehat{\psi}_{j}(\xi)=\widehat{\psi}\left(\xi / 2^{j}\right)$, the $L^{1}-L^{\infty}$ estimate yields

$$
\begin{aligned}
\left\|\psi_{-k} * \nabla f\right\|_{\infty} & \leq C_{n}\left\|\widehat{\psi}_{-k} \xi \hat{f}\right\|_{1} \\
& \leq C_{n} \int_{B_{2-k}}|\xi \hat{f}(\xi)| d \xi \\
& \leq C_{n}\left|B_{2^{-k}}\right|^{1 / 2}\left(\int_{B_{2-k}}|\xi|^{2}|\hat{f}(\xi)|^{2} d \xi\right)^{1 / 2} \\
& \leq C_{n} 2^{-k n}\|\nabla f\|_{2} \rightarrow 0
\end{aligned}
$$

as $k \rightarrow \infty$. Hence for a sufficiently large $k$ such that $\left\|\psi_{-k} * \nabla f\right\|_{\infty} \leq 1$, it suffices to estimate $\sum_{j \geq-k} \phi_{j} * f$. We apply the inequality (2.2) with small $\kappa$ specified below. For small $\kappa>0$ and 
$\alpha>0$ with $\kappa<\alpha<1-n / q$,

$$
\begin{aligned}
\left\|\nabla f_{+}\right\|_{\dot{F}_{\infty, 2}^{\kappa}} & =\left\|\left(\sum_{j=1}^{\infty} 2^{2 j \kappa}\left|\phi_{j} * \nabla f\right|^{2}\right)^{1 / 2}\right\|_{\infty} \\
& \leq\left(\sum_{j=1}^{\infty} 2^{2 j(\kappa-\alpha)}\right)^{1 / 2}\left\|\sup _{j} 2^{\alpha j}\left|\phi_{j} * \nabla f\right|\right\|_{\infty} \\
& \leq C\|\nabla f\|_{\dot{B}_{\infty, \infty}^{\alpha}} \leq C\|\nabla f\|_{\dot{B}_{q, \infty}^{\alpha+n / q}} \\
& \leq C\|\nabla f\|_{\dot{W}^{1, q}},
\end{aligned}
$$

where $\|\cdot\|_{W^{1, q}}$ stands for the homogeneous Sobolev semi-norm. This is possible under the condition $n<q$. On the other hand, using the $L^{\infty}$ boundedness of the Hardy-Littlewood maximal function (cf. Stein [36], p. 62-63), we have for small $0<\kappa<1$,

$$
\begin{aligned}
\left\|\nabla f_{-}\right\|_{\dot{F}_{\infty, 2}^{-\kappa}} & \leq\left\|\left(\sum_{j=-1}^{-\infty} 2^{2 j(1-\kappa)}\left|(\nabla \phi)_{j} * f\right|^{2}\right)^{1 / 2}\right\|_{\infty} \\
& \leq\left(\sum_{j=-1}^{-\infty} 2^{2 j(1-\kappa)}\right)^{1 / 2}\left\|\sup _{j}\left|(\nabla \phi)_{j} * f\right|\right\|_{\infty} \\
& \leq C\|M[f]\|_{\infty} \leq C\|f\|_{\infty},
\end{aligned}
$$

where $(\nabla \phi)_{j}(x)=2^{n j} \nabla \phi\left(2^{j} x\right)$ and $M[f]=\sup _{R, x} \frac{1}{\left|B_{R}\right|} \int_{B_{R}(x)}|f(y)| d y$ denotes the maximal function of $f$. From (2.4) and (2.5), we obtain the last inequality (2.3).

Theorem 2.4. Let $M$ be either $\mathbb{T}^{n}$ or $\mathbb{S}^{m}$. If $f: M \rightarrow \mathbb{S}^{m}$ such that $\nabla f \in W^{1, q}\left(M ; \mathbb{R}^{m+1}\right)$ for $n<q$, we have

$$
\|\nabla f\|_{\infty} \leq C(q)\left(1+\left(\|\nabla f\|_{B M O_{r}(M)}+1\right)\left(\log \left(e+\|\nabla f\|_{W^{1, q}}\right)\right)^{1 / 2}\right) .
$$

Remark. As is commented before, the inequality is the sharper version of the Brezis-Gallouet inequality (1.9) in $\Omega \subset \mathbb{R}^{2}$ because of the embedding inequality

$$
\|\nabla f\|_{B M O_{r}(\Omega)} \leq C(|\Omega|)\|\Delta f\|_{L^{2}(\Omega)} .
$$

Proof of Theorem 2.4. The proof is mostly the same in view of Proposition 2.3. We give the proof only in the case when $M=\mathbb{T}^{n}$. Let $\left\{\mathcal{K}_{k}\right\}_{k \in \mathbb{N}}$ be a finite covering over $\mathbb{T}^{n}$ with each radius $r_{0}$ and $\left\{\varphi_{k}\right\}$ be the partition of the unity such as

$$
\varphi_{k}= \begin{cases}1, & \mathcal{K}_{k}, \\ 0, & \left(\mathcal{K}_{k}\right)_{r_{0}}^{c}\end{cases}
$$


Then for $f: \mathbb{T}^{n} \rightarrow \mathbb{S}^{m}$ with $\nabla f \in W^{1, q}\left(\mathbb{T}^{n} ; \mathbb{S}^{m}\right)$, we apply Proposition 2.3 to $\varphi_{k} f$ as a function over $\mathbb{R}^{n}$;

$$
\begin{aligned}
\|\nabla f\|_{L^{\infty}\left(\mathcal{K}_{k}\right)} & \leq\left\|\nabla\left(\varphi_{k} f\right)\right\|_{\infty} \\
& \leq C(q)\left(1+\left\|\nabla\left(\varphi_{k} f\right)\right\|_{B M O}\left(\log \left(e+\left\|\nabla\left(\varphi_{k} f\right)\right\|_{W^{1, q}}\right)\right)^{1 / 2}\right) \\
& \leq C\left(q, \varphi_{k}\right)\left(1+\left(\|\nabla f\|_{B M O_{r}}+\|f\|_{\infty}\right)\left(\log \left(e+\|f\|_{W^{1, q}\left(\mathcal{K}_{k}\right)}+\|\nabla f\|_{W^{1, q}}\right)\right)^{1 / 2}\right) \\
& \leq C\left(q, \varphi_{k}\right)\left(1+\left(\|\nabla f\|_{B M O_{r}}+1\right)\left(\log \left(e+\|\nabla f\|_{W^{1, q}}\right)\right)^{1 / 2}\right) .
\end{aligned}
$$

Gathering the covering over $\mathbb{T}^{n}$, we concluded the inequality.

\section{The Regularity Criterion for the Smooth Solutions}

In this section, we give the proof of the regularity criterion to the weak solution of the harmonic heat flow. For simplicity, we consider only the flow from $\mathbb{R}^{2}$ onto sphere.

$$
\left\{\begin{aligned}
\partial_{t} u-\Delta u & =u(\nabla u, \nabla u), \quad t>0, x \in \mathbb{R}^{2}, \\
u(t, x) & : \mathbb{R}_{+} \times \mathbb{R}^{2} \rightarrow \mathbb{S}^{m} \\
u(0, x) & =u_{0}(x)
\end{aligned}\right.
$$

where $u(\nabla u, \nabla u)=u_{i} \sum_{1 \leq l, j \leq n} \nabla_{x_{k}} u_{i} \nabla_{x_{l}} u_{j}$ denotes the second fundamental form on the sphere and in the following we express this form as $u|\nabla u|^{2}$ unless it may cause any confusion. Before proving the main result Theorem 1.1, we state the smoothly prolonged result beyond the limiting time for the smooth solution, which was basically shown in [27].

Proposition 3.1 (Limiting regularity criterion [27]). Let $u$ be a smooth solution to (3.1) in $C\left([0, T) ; W^{1, \infty}\left(\mathbb{R}^{2} ; \mathbb{S}^{m}\right)\right) \cap C^{1}\left((0, T) ; W^{2, \infty}\left(\mathbb{R}^{2} ; \mathbb{S}^{m}\right)\right)$ with initial data $u_{0} \in W^{1, \infty}\left(\mathbb{R}^{2} ; \mathbb{S}^{m}\right)$. Suppose that the solution u satisfies

$$
\int_{0}^{T}\|\nabla u(\tau)\|_{B M O_{r}\left(\mathbb{R}^{2}\right)}^{2} d \tau<\infty
$$

Then the solution can be extended after $t=T$ namely, for some $\tilde{T}>T$, $u \in C\left([0, \tilde{T}) ; W^{1, \infty}\left(\mathbb{R}^{2} ; \mathbb{S}^{m}\right)\right) \cap C^{1}\left((0, T) ; W^{2, \infty}\left(\mathbb{R}^{2} ; \mathbb{S}^{m}\right)\right)$. In other word, if the solution blows up at $t=T$, then

$$
\int_{0}^{T}\|\nabla u(\tau)\|_{B M O_{r}\left(\mathbb{R}^{2}\right)}^{2} d \tau=\infty
$$

The proof is in fact a simple application of the argument in the previous section.

Proof of Proposition 3.1. Let $u$ be a smooth solution to (3.1) on $[0, T)$. By operating the Laplacian to the equation and then taking a $L^{2}$ inner product $(\cdot, \cdot)$ over $\mathbb{R}^{2}$ of the equation with 
$|\Delta u|^{q-2} \Delta u$, we have

$$
\begin{aligned}
\frac{1}{q} \frac{d}{d t}\|\Delta u(t)\|_{q}^{q}+ & \int_{\mathbb{T}^{n}} \nabla_{k} \Delta u(t) \cdot \nabla_{k}\left(|\Delta u|^{q-2} \Delta u(t)\right) d x \\
= & \left(|\nabla u(t)|^{2},|\Delta u(t)|^{q}\right) \\
& +2\left(\nabla_{k} u(t)\left(\nabla_{l} u(t) \cdot \nabla_{k} \nabla_{l} u(t)\right),|\Delta u(t)|^{q-2} \Delta u(t)\right) \\
& -2\left(u(t)\left(\nabla_{l} u(t) \cdot \nabla_{k} \nabla_{l} u(t)\right),|\Delta u(t)|^{q-2} \nabla_{k} \Delta u(t)\right) \\
& -2\left(u(t)\left(\nabla_{l} u(t) \cdot \nabla_{k} \nabla_{l} u(t)\right), \Delta u(t) \nabla_{k}\left(|\Delta u(t)|^{q-2}\right)\right) \\
\equiv & I_{1}+I_{2}+I_{3}+I_{4} .
\end{aligned}
$$

The first and second terms $I_{1}, I_{2}$ in (3.3) is dominated by the the elliptic estimate in $L^{q}$ (cf. [21]),

$$
I_{1}+I_{2} \leq\|\nabla u\|_{\infty}^{2}\|\Delta u\|_{q}^{q}
$$

For the third term $I_{3}$, we again use the elliptic estimate to have

$$
\begin{aligned}
I_{3} & \leq\|u\|_{\infty} \int_{\mathbb{R}^{2}}|\nabla u|\left|\nabla_{k} \nabla_{l} u\right| \cdot|\Delta u|^{q-2}\left|\nabla_{k} \Delta u\right| d x \\
& \leq C \int_{\mathbb{R}^{2}}|\nabla u|^{2}|\Delta u|^{q-2}\left|\nabla_{k} \nabla_{l} u\right|^{2} d x+\varepsilon \int_{\mathbb{R}^{2}}|\Delta u|^{q-2}\left|\nabla_{k} \Delta u\right|^{2} d x \\
& \leq C\|\nabla u\|_{\infty}^{2}\|\Delta u\|_{q}^{q}+\frac{1}{2} \int_{\mathbb{R}^{2}}|\Delta u|^{q-2}\left|\nabla_{k} \Delta u\right|^{2} d x .
\end{aligned}
$$

The last term $I_{4}$ can be dealt with a similar manner.

$$
I_{4}=\frac{q-2}{2} \int_{\mathbb{R}^{2}} u_{i} \nabla_{l} u_{j} \nabla_{k} \nabla_{l} u_{j} \Delta u_{i}|\Delta u|^{q-4} \nabla_{k}\left(|\Delta u|^{2}\right) d x
$$

$$
\leq C\|\nabla u\|_{\infty}^{2}\|\Delta u\|_{q}^{q}+\frac{1}{2} \int_{\mathbb{R}^{2}}|\Delta u|^{q-2}\left|\nabla_{k} \Delta u\right|^{2} d x .
$$

On the other hand, the second term in the left hand side of (3.3) is

$$
\begin{aligned}
& \int_{\mathbb{R}^{2}} \nabla_{k} \Delta u \cdot \nabla_{k}\left(|\Delta u|^{q-2} \Delta u\right) d x \\
& \quad=\int_{\mathbb{R}^{2}}|\Delta u|^{q-2}\left|\nabla_{k} \Delta u\right|^{2} d x+\left.\left.\frac{q-2}{4} \int_{\mathbb{R}^{2}}\left|\left(|\Delta u|^{2}\right)^{(q-4) / 4} \nabla_{k}\right| \Delta u\right|^{2}\right|^{2} d x \\
& =\int_{\mathbb{R}^{2}}|\Delta u|^{q-2}\left|\nabla_{k} \Delta u\right|^{2} d x+\frac{4(q-2)}{q^{2}} \int_{\mathbb{R}^{2}}\left|\nabla\left(|\Delta u|^{2}\right)^{q / 4}\right|^{2} d x .
\end{aligned}
$$

Hence by gathering estimates (3.4)-(3.6) and (3.7) and plug into (3.3), it follows

$$
\begin{gathered}
\frac{1}{q} \frac{d}{d t}\|\Delta u(t)\|_{q}^{q}+\frac{4(q-2)}{q^{2}}\left\|\nabla|\Delta u|^{q / 2}\right\|_{2}^{2} \\
\leq C\|\nabla u\|_{\infty}^{2}\|\Delta u\|_{q}^{q} .
\end{gathered}
$$

Integration (3.8) over $[0, T]$ and the Young inequality implies

$$
\|\Delta u(t)\|_{q}^{q} \leq\|\Delta u(0)\|_{q}^{q}+C(\varepsilon) \int_{0}^{T}\|\nabla u\|_{\infty}^{2}\|\Delta u(\tau)\|_{q}^{q} d \tau
$$


Noting the energy inequality (1.3), the logarithmic inequality (2.3) in Corollary 2.4 yields that for $\gamma>n / q$ and $q>n$

$$
\|\nabla u\|_{\infty} \leq C\left(1+\left(1+\|\nabla u\|_{B M O}\right)\left(\log \left(e+\|\nabla u\|_{W^{1, q}}\right)\right)^{1 / 2}\right) .
$$

Hence it follows from (3.9) and (3.10)

$$
\|\Delta u(t)\|_{q}^{q} \leq\left\|\Delta u_{0}\right\|_{q}^{q}+C \int_{0}^{T}\|\nabla u(\tau)\|_{B M O}^{2}\left(1+\log \left(e+\|\nabla u(\tau)\|_{W^{1, q}}\right)^{\frac{1}{2}}\right)^{2}\|\Delta u(\tau)\|_{q}^{q} d \tau .
$$

Combining with the energy inequality

$$
\|\nabla u(t)\|_{2}^{2}+2 \int_{0}^{t}\left\|\partial_{t} u(\tau)\right\|_{2}^{2} d \tau \leq\left\|\nabla u_{0}\right\|_{2}^{2}
$$

and $\|u\|_{\infty}=1$, we conclude by the Gronwall argument that

$$
\|\nabla u(t)\|_{W^{1, q}}^{q} \leq C\left\|\nabla u_{0}\right\|_{W^{1, q}}^{q} \exp \left\{C \exp \left(C \int_{0}^{T}\left(1+\|\nabla u(\tau)\|_{B M O}^{2}\right) d \tau\right)\right\} .
$$

This estimate assures that the solution has the regularity in $C\left((0, T] ; \dot{W}^{2, q}\right)$ under the assumption (3.2). Since we have chosen that $q>n$, the Sobolev embedding implies that $\nabla u(t)$ is a continuous function in $(x, t)$. A general argument for the harmonic heat flow gives the higher regularity. This completes the proof of Proposition 3.1.

Remark. There is an another method to make continuation of the smooth solution after the maximal existence time. According to Struwe [37], there exist $\delta>0$ and $\varepsilon>0$ such that if for any $R$ with $\delta R^{2}<T$ there holds

$$
\inf _{T-\delta R^{2}<t<T} \sup _{x} \int_{B_{r}(x)}|\nabla u(t)|^{2} d x<\varepsilon
$$

then the solution $u$ in $[0, T)$ can be prolonged smoothly after $t=T$. If we use this criterion, we can derive the same conclusion as in Proposition 3.1 under the same assumption (3.2). Indeed, our monotonicity for the mean oscialltion provides the above smallness condition under the regularity assumption (3.2).

\section{Monotonicity By meAn oscillation}

In this section, we prove that the monotonicity formula and $\varepsilon$-regularity theorem by mean oscillation hold for a smooth solution to (1.2).

Theorem 4.1. Let $u$ be a smooth solution of (1.2). For any fixed $\delta>0, T>0$ and $r>0$ we set a time interval $I_{\delta^{1 / 2} r}(T)=\left(T-\delta r^{2}, T\right)$. Then for any $x_{0} \in \mathbb{R}^{2}$, there exists an absolute constant $C>0$ such that for any $r \in(0, R)$, we have

$$
\begin{aligned}
\int_{I_{\delta^{1 / 2} r}(T)} & \left(\frac{1}{\pi r^{2}} \int_{B_{r}\left(x_{0}\right)}\left|\nabla u(\tau)-\overline{\nabla u}_{B_{r}\left(x_{0}\right)}(\tau)\right|^{2} d x\right) d \tau \\
\leq & \int_{I_{\delta^{1 / 2} R(T)}}\left(\frac{1}{\pi R^{2}} \int_{B_{R}\left(x_{0}\right)}\left|\nabla u(\tau)-\overline{\nabla u}_{B_{R}}(\tau)\right|^{2} d x\right) d \tau+C \delta E\left(u_{0}\right),
\end{aligned}
$$

where $B_{R}\left(x_{0}\right)=\left\{\left|x-x_{0}\right|<R\right\}$. 
Proof of Theorem 4.1. Let $u(t, x)$ be a smooth solution of (1.2) and $T>0$ be an arbitrary fixed time. For any fixed $x_{0} \in \mathbb{R}^{2}$, we choose a test fuction $\eta(x) \in C_{0}^{\infty}\left(\mathbb{R}^{2} ; \mathbb{R}\right)$ such that $0 \leq \eta \leq 1, \eta=1$ in $B_{R / 2}\left(x_{0}\right)$, supp $\eta \in B_{R}\left(x_{0}\right)$ and $|\nabla \eta| \leq 4 / R$ in $\mathbb{R}^{2}$. Letting

$$
\widetilde{\nabla u}(t) \equiv \frac{1}{\|\eta\|_{2}^{2}} \int_{\mathbb{R}^{2}} \eta^{2} \nabla u(t) d x
$$

and multiplying the equation (1.2) by $\eta^{2} \partial_{t} u$, we see from $\partial_{t}|u|^{2}=0$ that

$$
\begin{aligned}
\left|\partial_{t} u\right|^{2} \eta^{2} & -\operatorname{div}\left((\nabla u-\widetilde{\nabla u}) \cdot \partial_{t} u \eta^{2}\right) \\
& +(\nabla u-\widetilde{\nabla u}) \cdot \partial_{t} \nabla u \eta^{2}+2 \partial_{t} u \cdot(\nabla u-\widetilde{\nabla u}) \cdot \nabla \eta \eta=0
\end{aligned}
$$

Integrating (4.2) over $(s, t) \times \mathbb{R}^{2}$, we have, by the divergence theorem,

$$
\int_{s}^{t} \int_{\mathbb{R}^{2}}\left(\left|\partial_{t} u\right|^{2} \eta^{2}+\eta^{2}(\nabla u-\widetilde{\nabla u}) \cdot \partial_{t} \nabla u+2 \partial_{t} u \cdot(\nabla u-\widetilde{\nabla u}) \cdot \nabla \eta \eta\right) d x d \tau=0 .
$$

The second term of the left hand side of (4.3) can be treated as

$$
\begin{aligned}
\int_{s}^{t} \int_{\mathbb{R}^{2}} & \eta^{2} \partial_{t} \nabla u \cdot(\nabla u-\widetilde{\nabla u}) d x d \tau \\
= & \frac{1}{2} \int_{s}^{t} \frac{d}{d t} \int_{\mathbb{R}^{2}}|\nabla u-\widetilde{\nabla u}|^{2} \eta^{2} d x d \tau+\int_{s}^{t} \int_{\mathbb{R}^{2}} \eta^{2} \partial_{t} \widetilde{\nabla u} \cdot(\nabla u-\overline{\nabla u}) d x d \tau \\
= & \frac{1}{2} \int_{\mathbb{R}^{2}}|\nabla u(t)-\widetilde{\nabla u}(t)|^{2} \eta^{2} d x-\frac{1}{2} \int_{\mathbb{R}^{2}}|\nabla u(s)-\widetilde{\nabla u}(s)|^{2} \eta^{2} d x \\
& +\int_{s}^{t} \int_{\mathbb{R}^{2}} \eta^{2} \partial_{t} \widetilde{\nabla u} \cdot(\nabla u-\widetilde{\nabla u}) d x d \tau
\end{aligned}
$$

Since

$$
\begin{aligned}
& \int_{s}^{t} \int_{\mathbb{R}^{2}} \eta^{2} \partial_{t} \widetilde{\nabla u} \cdot(\nabla u-\widetilde{\nabla u}) d x d \tau \\
& =\int_{s}^{t} \partial_{t} \widetilde{\nabla u}(\tau) \cdot \int_{\mathbb{R}^{2}}(\nabla u-\widetilde{\nabla u}) \eta^{2} d x d \tau \\
& =\int_{s}^{t} \partial_{t} \widetilde{\nabla u}(\tau) \cdot\left(\int_{\mathbb{R}^{2}} \eta^{2} \nabla u(\tau) d x-\widetilde{\nabla u}(\tau) \int_{\mathbb{R}^{2}} \eta^{2} d x\right) d \tau \\
& =0
\end{aligned}
$$

we obtain from (4.3) that

$$
\begin{gathered}
\int_{s}^{t} \int_{\mathbb{R}^{2}}\left|\partial_{t} u\right|^{2} \eta^{2} d x d \tau+\frac{1}{2} \int_{\mathbb{R}^{2}}|\nabla u(t)-\widetilde{\nabla u}(t)|^{2} \eta^{2} d x-\frac{1}{2} \int_{\mathbb{R}^{2}}|\nabla u(s)-\widetilde{\nabla u}(s)|^{2} \eta^{2} d x \\
=-2 \int_{s}^{t} \int_{\mathbb{R}^{2}} \partial_{t} u \cdot(\nabla u-\widetilde{\nabla u}) \cdot \nabla \eta \eta d x d \tau .
\end{gathered}
$$

The right hand side of (4.4) is estimated by

$$
\begin{aligned}
& 2 \int_{s}^{t} \int_{\mathbb{R}^{2}}\left|\nabla u-\widetilde{\nabla u}(\tau)\left\|\partial_{t} u\right\| \eta \nabla \eta\right| d x d \tau \\
\leq & \frac{1}{2} \int_{s}^{t}\left|\partial_{t} u\right|^{2} \eta^{2} d x d \tau+2 \int_{s}^{t} \int_{\mathbb{R}^{2}}|\nabla u-\widetilde{\nabla u}|^{2} \eta^{2}|\nabla \eta|^{2} d x d \tau
\end{aligned}
$$


and it follows from (4.4) that

$$
\begin{aligned}
& \int_{\mathbb{R}^{2}}|\nabla u(t)-\widetilde{\nabla u}(t)|^{2} \eta^{2} d x-\int_{\mathbb{R}^{2}}|\nabla u(s)-\widetilde{\nabla u}(s)|^{2} \eta^{2} d x \\
& \quad+\int_{s}^{t} \int_{\mathbb{R}^{2}}\left|\partial_{t} u\right|^{2} \eta^{2} d x d \tau \\
& \leq 4 \int_{s}^{t} \int_{\mathbb{R}^{2}}|\nabla u-\widetilde{\nabla u}|^{2}|\eta \nabla \eta|^{2} d x d \tau \\
& \leq \frac{64}{R^{2}} \int_{s}^{t} \int_{\mathbb{R}^{2}}\left(|\nabla u|^{2}-|\widetilde{\nabla u}|^{2}\right)|\eta|^{2} d x d \tau \\
& \leq \frac{64}{R^{2}} \int_{s}^{t} \int_{\mathbb{R}^{2}}|\nabla u|^{2} \eta^{2} d x \leq 64 E\left(u_{0}\right) \frac{(t-s)}{R^{2}} .
\end{aligned}
$$

Let $\delta>0$ be arbitrary fixed and $r \in(0, R / 2)$. Then for $s \in I_{\delta^{1 / 2} R}(T) \backslash I_{\delta^{1 / 2} r}(T)$ and $t \in I_{\delta^{1 / 2} r}(T)$, we see by taking the integral avearge of (4.5) by $s$ over $I_{\delta^{1 / 2} R}(T) \backslash I_{\delta^{1 / 2} r}(T)$,

$$
\begin{aligned}
& \int_{\mathbb{R}^{2}}|\nabla u(t)-\widetilde{\nabla u}(t)|^{2} \eta^{2} d x \\
& \leq \frac{1}{\left|I_{\delta^{1 / 2} R}(T) \backslash I_{\delta^{1 / 2} r}(T)\right|} \int_{I_{\delta^{1 / 2} R_{R}}(T) \backslash I_{\delta^{1 / 2}{ }_{r}}(T)}|\nabla u(s)-\widetilde{\nabla u}(s)|^{2} d x d s \\
& \quad+64 E\left(u_{0}\right) \delta,
\end{aligned}
$$

where we use that $|t-s| \leq \delta R^{2}$. Again by integrating (4.6) by $t \in I_{\delta^{1 / 2} r}(T)$, we see

$$
\begin{aligned}
\int_{I_{\delta^{1 / 2}}(T)} & \int_{\mathbb{R}^{2}}|\nabla u(\tau)-\widetilde{\nabla u}(\tau)|^{2} \eta^{2} d x d \tau \\
\leq & \frac{\left|I_{\delta^{1 / 2} r}(T)\right|}{\left|I_{\delta^{1 / 2} R}(T) \backslash I_{\delta^{1 / 2} r}(T)\right|} \int_{I_{\delta^{1 / 2} R_{R}}(T) \backslash I_{\delta^{1 / 2} r}(T)} \int_{\mathbb{R}^{2}}|\nabla u(\tau)-\widetilde{\nabla u}(\tau)|^{2} d x d \tau \\
& +64 E\left(u_{0}\right) \delta^{2} r^{2} \\
\leq & \frac{r^{2}}{R^{2}-r^{2}} \int_{I_{\delta^{1 / 2} R_{R}}(T) \backslash I_{\delta^{1 / 2} r_{r}}(T)} \int_{\mathbb{R}^{2}}|\nabla u(\tau)-\widetilde{\nabla u}(\tau)|^{2} d x d \tau \\
& +64 E\left(u_{0}\right) \delta^{2} r^{2}
\end{aligned}
$$

Adding

$$
\frac{r^{2}}{R^{2}-r^{2}} \int_{I_{\delta^{1 / 2} r}(T)} \int_{\mathbb{R}^{2}}|\nabla u(\tau)-\widetilde{\nabla u}(\tau)|^{2} \eta d x d \tau
$$

to the both side of (4.7), we have

$$
\begin{aligned}
& \frac{R^{2}}{R^{2}-r^{2}} \int_{I_{\delta^{1 / 2} r}(T)} \int_{\mathbb{R}^{2}}|\nabla u(\tau)-\widetilde{\nabla u}(\tau)|^{2} \eta^{2} d x d \tau \\
& \leq \frac{r^{2}}{R^{2}-r^{2}} \int_{I_{\delta^{1 / 2} R}(T)} \int_{\mathbb{R}^{2}}|\nabla u(\tau)-\widetilde{\nabla u}(\tau)|^{2} \eta^{2} d x d \tau+64 E\left(u_{0}\right) \delta^{2} r^{2} .
\end{aligned}
$$

Therefore we obtain from (4.8) that

$$
\begin{aligned}
\int_{I_{\delta^{1 / 2} /}(T)} & \frac{1}{\pi r^{2}} \int_{\mathbb{R}^{2}}|\nabla u(\tau)-\widetilde{\nabla u}(\tau)|^{2} \eta^{2} d x d \tau \\
& \leq \int_{I_{\delta^{1 / 2} R}(T)} \frac{1}{\pi R^{2}} \int_{\mathbb{R}^{2}}|\nabla u(\tau)-\widetilde{\nabla u}(\tau)|^{2} \eta^{2} d x d \tau+\frac{64 E\left(u_{0}\right)}{\pi} \delta^{2} .
\end{aligned}
$$


Finally, we remove the cut off function $\eta$. Make the routine calculation to have, for all $\tau \in I_{\delta^{1 / 2} R}$,

$$
\begin{aligned}
& \int_{\mathbb{R}^{2}}|\nabla u(\tau)-\widetilde{\nabla u}(\tau)|^{2} \eta^{2} d x \\
& \quad=\int_{\mathbb{R}^{2}}\left|\nabla u(\tau)-\overline{\nabla u}_{B_{R}\left(x_{0}\right)}(\tau)-\left(\widetilde{\nabla u}(\tau)-\overline{\nabla u}_{B_{R}\left(x_{0}\right)}(\tau)\right)\right|^{2} \eta^{2} d x \\
& \quad=\int_{\mathbb{R}^{2}}\left|\nabla u(\tau)-\overline{\nabla u}_{B_{R}\left(x_{0}\right)}(\tau)\right|^{2} \eta^{2} d x-\left(\int_{\mathbb{R}^{2}} \eta^{2} d x\right)\left|\widetilde{\nabla u}(\tau)-\overline{\nabla u}_{B_{R}\left(x_{0}\right)}(\tau)\right|^{2} \\
& \leq \int_{B_{R}\left(x_{0}\right)}\left|\nabla u(\tau)-\overline{\nabla u}_{B_{R}\left(x_{0}\right)}(\tau)\right|^{2} d x
\end{aligned}
$$

While it holds for any $r \in(0, R / 2)$ and $\tau \in I_{\delta^{1 / 2} r}$ that

$$
\begin{aligned}
& \int_{\mathbb{R}^{2}}|\nabla u(\tau)-\widetilde{\nabla u}(\tau)|^{2} \eta^{2} d x \\
& \quad \geq \int_{B_{R / 2}\left(x_{0}\right)}|\nabla u(\tau)-\widetilde{\nabla u}(\tau)|^{2} d x \\
& \quad \geq \int_{B_{r}\left(x_{0}\right)}\left|\nabla u(\tau)-\overline{\nabla u}_{B_{r}\left(x_{0}\right)}(\tau)\right|^{2} d x .
\end{aligned}
$$

Substituting (4.10) and (4.11) into (4.9), we obtain our conclusion.

Under the smallness hypothesis of the mean oscillation, we can prove the local regularity. We need the following general inequality which is a variant of well-known Nash's inequality:

Lemma 4.2. Let $\phi(t, x)$ be a positive cut off function supported in $(-1,1) \times B_{1}$ with $\phi(t, x) \equiv 1$ over $(-1 / 2,1 / 2) \times B_{1 / 2}$ and $0 \leq \phi \leq 1$. Then for $\nabla f \in L^{\infty}\left(0, T ; L^{2}\left(\mathbb{R}^{2}\right)\right) \cap L^{2}\left(0, T ; H^{1}\left(\mathbb{R}^{2}\right)\right)$, there exists a constant $C>0$ such that

$$
\begin{aligned}
& \left(\int_{I_{1 / 2}} \int_{B_{1 / 2}}|\nabla f|^{4} d x d t\right)^{2} \\
& \leq\left(\sup _{t \in I} \int_{B_{1}}|\nabla f|^{2} d x\right)^{2}\left\{\left.\left.\int_{I_{1}} \int_{B_{1}}|\nabla| \nabla f\right|^{2}\right|^{2} \phi^{4} d x d t+\int_{I_{1}} \int_{B_{1}}|\nabla f|^{4}\left|\nabla \phi^{2}\right|^{2} d x d t\right\},
\end{aligned}
$$

where $I_{1 / 2}=(-1 / 2,1 / 2)$ and $B_{1 / 2}=\left\{x \in \mathbb{R}^{2},|x|<1 / 2\right\}$.

Proof of Lemma 4.2. By well known Nash's inequality:

$$
\|g\|_{2}^{2} \leq \frac{1}{2 \pi}\|g\|_{1}\|\nabla g\|_{2}, \quad g \in H^{1}\left(\mathbb{R}^{2}\right) \cap L^{1}\left(\mathbb{R}^{2}\right),
$$

we have for $\phi^{2}|\nabla f|^{2}$,

$$
\int_{B_{1}}|\nabla f|^{4} \phi^{4} d x \leq \frac{1}{2 \pi}\left(\int_{B_{1}}|\nabla f|^{2} \phi^{2} d x\right)\left(\int_{B_{1}}\left(\left.\left.|\nabla| \nabla f\right|^{2}\right|^{2} \phi^{4}+|\nabla f|^{4}\left|\nabla \phi^{2}\right|^{2}\right) d x\right)^{1 / 2} .
$$


Thus we have

$$
\begin{aligned}
\int_{I_{1 / 2}} & \int_{B_{1 / 2}}|\nabla f|^{4} d x d t \\
\leq & \frac{1}{\pi}\left(\sup _{t \in I} \int_{B_{1}}|\nabla f(t)|^{2} d x\right) \\
& \times\left\{\left(\left.\left.\int_{I} \int_{B_{1}}|\nabla| \nabla f\right|^{2}\right|^{2} \phi^{4} d x d t\right)^{1 / 2}+\left(\int_{I} \int_{B_{1}}|\nabla f|^{4}\left|\nabla \phi^{2}\right|^{2} d x d t\right)^{1 / 2}\right\} .
\end{aligned}
$$

The desired estimate follows directly from (4.14).

Following the analogous argument as in Schoen [33], we have the following $\varepsilon$-regularity criterion for the harmonic heat flow (1.2).

Theorem 4.3. Let $u$ be a smooth solution of the harmonic heat flow (1.2). Then there exist absolute constants $\varepsilon_{0}>0$ and $R_{0}>0$ such that if

$$
\int_{I_{R}\left(t_{0}\right)} \frac{1}{R^{2}} \int_{B_{R}\left(x_{0}\right)}\left|\nabla u(t)-\overline{\nabla u}_{B_{R}}(t)\right|^{2} d x d t \leq \varepsilon_{0}
$$

holds for all $R \in\left(0, R_{0}\right)$, then

$$
\|\nabla u\|_{L^{\infty}\left(I_{R_{0} / 2}\left(t_{0}\right) \times B_{R_{0} / 2}\left(x_{0}\right)\right)} \leq \frac{C}{\left(R_{0}\right)^{2}},
$$

where $I_{R}\left(t_{0}\right)=\left(t_{0}-R^{2}, t_{0}\right)$ and $B_{R}\left(x_{0}\right)=\left\{x \in \mathbb{R}^{2} ;\left|x-x_{0}\right|<R\right\}$.

Invoking the monotonicity formula, Theorem 4.1, we find that the assumption (4.15) in Theorem 4.3 can be relaxed into the one for some $R>0$, and thus, we have the following criterion for the singularity:

Corollary 4.4. Let $u$ be a smooth solution of the harmonic heat flow (1.2) in $\left(0, t_{0}\right) \times \mathbb{R}^{2}$. Then there exists an absolute constant $\varepsilon_{0}>0$ such that we have for all $R>0$

$$
\int_{I_{R}\left(t_{0}\right)} \frac{1}{R^{2}} \int_{B_{R}\left(x_{0}\right)}\left|\nabla u(t)-\overline{\nabla u}_{B_{R}}(t)\right|^{2} d x d t \geq \varepsilon_{0}
$$

whenever $\left(t_{0}, x_{0}\right)$ is a singularity of the solution.

Proof of Theorem 4.3. For a point $\left(t_{0}, x_{0}\right)$ we define a parabolic neighborhood around $\left(t_{0}, x_{0}\right)$ by $P_{R} \equiv P_{R}\left(t_{0}, x_{0}\right)=I_{R}\left(t_{0}\right) \times B_{R}\left(x_{0}\right)$ for $R>0$.

Let $u(t . x)$ be a smooth solution to (1.2) and $R_{0}$ be the scaling parameter which assures the estimate (4.15). Then we choose a number $\sigma_{0}$ such that it attains the maximum of

$$
F(r)=\left(R_{0}-r\right)^{2} \sup _{(t, x) \in P_{r}}|\nabla u(t, x)|^{2}
$$

over $r \in\left(0, R_{0}\right]$. If $\sigma_{0}=R_{0}$ then the result is obvious since $F\left(R_{0}\right)=0$. Hence $\sigma \in\left(0, R_{0}\right)$.

Changing the parabolic neighborhood $P_{\sigma_{0}}$ smaller if necessary, we choose $\left(\tilde{t}_{0}, \tilde{x}_{0}\right) \in \overline{P_{\sigma_{0}}}$ so that it attains the maximum of $|\nabla u(t, x)|$ over $\overline{P_{\sigma_{0}}}$, namely

$$
\left|\nabla u\left(\tilde{t}_{0}, \tilde{x}_{0}\right)\right|=\max _{P_{\sigma_{0}}}|\nabla u(t, x)| .
$$


Letting

$$
M \equiv\left|\nabla u\left(\tilde{t}_{0}, \tilde{x}_{0}\right)\right|, \quad \rho_{0} \equiv \frac{R_{0}-\sigma_{0}}{2},
$$

we find from (4.17) and the choice of $\sigma_{0}$ that

$$
\sup _{P_{\sigma_{0}+\rho_{0}}}|\nabla u(t, x)|^{2} \leq \sup _{P_{\sigma_{0}}}|\nabla u(t, x)|^{2} \leq 2^{2} M^{2} .
$$

We particularly choose $R=M^{-1}$ as the scaling parameter of the scaled solution $u_{R}(t, x)=$ $u\left(\tilde{t}_{0}+R^{2} t, \tilde{x}_{0}+R x\right)$. Then again $u_{R}$ solves (1.2) and by the choice of $M$, we have

$$
\left|\nabla u_{R}(0,0)\right|^{2}=1, \quad \sup _{P_{\sigma_{0}}}\left|\nabla u_{R}(t, x)\right|^{2} \leq 2^{2}
$$

when $R=M^{-1}$.

By differentiating the $k$ th component of the scaled equation (1.2) by $x_{i}$ and multiplying it by $\nabla_{i}\left(u_{R}\right)_{k}$ and making summation, we see

$$
\frac{1}{2} \partial_{t}\left|\nabla u_{R}\right|^{2}-\frac{1}{2} \Delta\left|\nabla u_{R}\right|^{2}+\left|\nabla \nabla u_{R}\right|^{2}=\left|\nabla u_{R}\right|^{4}+\left(u_{R}\right)_{k} \nabla_{i}\left(u_{R}\right)_{k} \nabla u_{R} \cdot \nabla_{i}\left(\nabla u_{R}\right) .
$$

The last term of (4.21) disappears since $\left|u_{R}\right|^{2}=1$.

We now claim that $\rho_{0} M \leq 1$. Assume on the contrary that $\rho_{0} M>1$. In this case, we can choose the fixed parabolic region $P_{1}(0)=I_{1}(0) \times B_{1}(0)$ such that $P_{1}(0) \subset P_{\rho_{0} M}(0)$. On this region, we proceed by the well known Moser's Harnack estimate for the scaled solution $u_{R}$.

Let $\phi(t, x)$ be a smooth function defined by $\phi(t, x)=\psi_{1}(t) \psi_{2}(x)$, where $\psi_{1}(t)$ is supported in $(-1,1)$ and $\psi_{1}(t) \equiv 1$ on $(-1 / 2,1 / 2)$ with $0 \leq \psi_{1}(t) \leq 1$ and $\psi_{2}(x)$ is supported in $B_{1}(0)$, $\psi_{2}(x) \equiv 1$ on $B_{1 / 2}(0)$ with $0 \leq \psi_{2}(x) \leq 1$. Note that

$$
|\nabla \phi| \leq 2, \quad|\Delta \phi| \leq 2^{2}, \quad\left|\partial_{t} \phi\right| \leq 2, \quad|\nabla \Delta \phi| \leq 2^{3} .
$$

Multiply (4.21) by $\phi^{4}\left|\nabla u_{R}\right|^{2}$ to have

$$
\begin{aligned}
& \frac{1}{2} \partial_{t} \int_{B_{1}}\left|\nabla u_{R}\right|^{4} \phi^{4} d x-\frac{1}{2} \int_{B_{1}}\left|\nabla u_{R}\right|^{4} \partial_{t} \phi^{4} d x \\
& \quad+\left.\left.\int_{B_{1}}|\nabla| \nabla u_{R}\right|^{2}\right|^{2} \phi^{4} d x+2 \int_{B_{1}}\left|\nabla u_{R}\right|^{2}\left|\nabla \nabla u_{R}\right|^{2} \phi^{4} d x+\int_{B_{1}}\left|\nabla u_{R}\right|^{2} \nabla\left|\nabla u_{R}\right|^{2} \cdot \nabla \phi^{4} d x \\
& =2 \int_{B_{1}}\left|\nabla u_{R}\right|^{6} \phi^{4} d x .
\end{aligned}
$$

Let $I_{r}=(-r, r)$. Applying Lemma 4.2 and integrating $(4.22)$ over $I_{1}=(-1,1)$ in $t$ yield the following estimate:

$$
\begin{aligned}
& \left(\int_{I_{1 / 2}} \int_{B_{1 / 2}}\left|\nabla u_{R}\right|^{4} d x d t\right)^{2}\left(\sup _{t \in I_{1}} \int_{B_{1}}\left|\nabla u_{R}\right|^{2} d x\right)^{-2}+\left.\left.\int_{I_{1}} \int_{B_{1}}|\nabla| \nabla u_{R}\right|^{2}\right|^{2} \phi^{4} d x d t \\
& \quad \leq\left.\left. 2 \int_{I_{1}} \int_{B_{1}}|\nabla| \nabla u_{R}\right|^{2}\right|^{2} \phi^{4} d x d t+\int_{I_{1}} \int_{B_{1}}\left|\nabla u_{R}\right|^{4}\left|\nabla \phi^{2}\right|^{2} d x d t \\
& \quad=4 \int_{I_{1}} \int_{B_{1}}\left|\nabla u_{R}\right|^{6} \phi^{4} d x d t+\int_{I_{1}} \int_{B_{1}}\left|\nabla u_{R}\right|^{4}\left(\Delta\left(\phi^{4}\right)+\partial_{t}\left(\phi^{4}\right)+\left|\nabla \phi^{2}\right|^{2}\right) d x d t \\
& \quad \equiv A+B .
\end{aligned}
$$

Here we have put the last two terms as $A$ and $B$. 
We use the scaled equation (1.2) and $\left|u_{R}\right|=1$ to have for $l=2,3$ that

$$
\begin{aligned}
\left|\nabla u_{R}\right|^{2 l}= & -\nabla_{i}\left(u_{R} \cdot\left(\nabla_{i} u_{R}-\bar{\nabla}_{i} u_{B_{R}}\right)\left|\nabla u_{R}\right|^{2(l-1)}\right)+\nabla_{i} u_{R} \cdot\left(\nabla_{i} u_{R}-\bar{\nabla}_{i} u_{B_{R}}\right)\left|\nabla u_{R}\right|^{2(l-1)} \\
& +u_{R} \cdot\left(\nabla_{i} u_{R}-\bar{\nabla}_{i} u_{B_{R}}\right)\left|\nabla u_{R}\right|^{2(l-2)} \nabla_{i}\left|\nabla u_{R}\right|^{2} .
\end{aligned}
$$

It follows from (4.24) with $l=3$, integration by parts, Hölder's and Cauchy's inequalities that the first term of the right hand side of (4.23) is estimated by

$$
\begin{aligned}
A \leq & \int_{I_{1}} \int_{B_{1}}\left|u_{R}\right|\left|\nabla u_{R}-\overline{\nabla u_{B_{R}}}\right|\left|\nabla u_{R}\right|^{4}\left|\nabla \phi^{4}\right| d x d t+\int_{I_{1}} \int_{B_{1}}\left|\nabla u_{R}-\overline{\nabla u_{B_{R}}}\right|\left|\nabla u_{R}\right|^{5} \phi^{4} d x d t \\
& +\left.4 \int_{I_{1}} \int_{B_{1}}\left|u_{R}\right|\left|\nabla u_{R}-\overline{\nabla u}_{B_{R}}\right|\left|\nabla u_{R}\right|^{2}|\nabla| \nabla u_{R}\right|^{2} \mid \phi^{4} d x d t \\
\leq & \left(\int_{I_{1}} \int_{B_{1}}\left|\nabla u_{R}-\overline{\nabla u}_{B_{R}}\right|^{2} d x d t\right)^{1 / 2} \\
& \times C(\phi)\left\{\left(\int_{I_{1}} \int_{B_{1}}\left|\nabla u_{R}\right|^{8} d x d t\right)^{1 / 2}+\left(\int_{I_{1}} \int_{B_{1}}\left|\nabla u_{R}\right|^{10} d x d t\right)^{1 / 2}\right\} \\
& +\left.\left.\frac{1}{4} \int_{I_{1}} \int_{B_{1}}|\nabla| \nabla u_{R}\right|^{2}\right|^{2} \phi^{4} d x d t \\
& +\left(\int_{I_{1}} \int_{B_{1}}\left|\nabla u_{R}-\overline{\nabla u}_{B_{R}}\right|^{4} d x d t\right)^{1 / 2}\left(\int_{I_{1}} \int_{B_{1}}\left|\nabla u_{R}\right|^{8} \phi^{8} d x d t\right)^{1 / 2} .
\end{aligned}
$$

An analogous application (4.24) with $l=2$ makes the second term in the right hand side of (4.23) bounded by

$$
\begin{aligned}
& B \leq \int_{I_{1}} \int_{B_{1}}\left|u_{R}\right|\left|\nabla u_{R}-\bar{\nabla}_{B_{R}}\right|\left|\nabla u_{R}\right|^{2}\left|\nabla\left(\Delta\left(\phi^{4}\right)+\partial_{t}\left(\phi^{4}\right)+\left|\nabla \phi^{2}\right|^{2}\right)\right| d x d t \\
& +\left.\int_{I_{1}} \int_{B_{1}}\left|\nabla u_{R}-\overline{\nabla u}_{B_{R}}\right|\left|\nabla u_{R}\right|^{3}\left|\Delta\left(\phi^{4}\right)+\partial_{t}\left(\phi^{4}\right)+\right| \nabla \phi^{2}\right|^{2} \mid d x d t \\
& +\left.8 \int_{I_{1}} \int_{B_{1}}\left|u_{R}\right|\left|\nabla u_{R}-\overline{\nabla u_{B_{R}}}{ }\right||\nabla| \nabla u_{R}\right|^{2}\left|\phi^{2}\right| \phi \Delta \phi+\phi \partial_{t} \phi+|\nabla \phi|^{2} \mid d x d t \\
& \leq C(\phi)\left(\int_{I_{1}} \int_{B_{1}}\left|\nabla u_{R}-\overline{\nabla u}_{B_{R}}\right|^{2} d x d t\right)^{1 / 2} \\
& \times\left\{\left(\int_{I_{1}} \int_{B_{1} \backslash B_{1 / 2}}\left|\nabla u_{R}\right|^{4} d x d t\right)^{1 / 2}+\left(\int_{I_{1}} \int_{B_{1} \backslash B_{1 / 2}}\left|\nabla u_{R}\right|^{6} d x d t\right)^{1 / 2}\right\} \\
& +\left.\left.\frac{1}{4} \int_{I_{1}} \int_{B_{1}}|\nabla| \nabla u_{R}\right|^{2}\right|^{2} \phi^{4} d x d t \\
& +C(\phi)\left\|u_{R}\right\|_{\infty} \int_{I_{1}} \int_{B_{1}}\left|\nabla u_{R}-\overline{\nabla u}_{B_{R}}\right|^{2} d x d t .
\end{aligned}
$$


Combining (4.25) and (4.26) with (4.23), we obtain

$$
\begin{gathered}
\left(\int_{I_{1 / 2}} \int_{B_{1 / 2}}\left|\nabla u_{R}\right|^{4} d x d t\right)^{2}\left(\sup _{t \in I} \int_{B_{1}}\left|\nabla u_{R}(t)\right|^{2} \phi d x\right)^{-2}+\left.\left.\frac{1}{2} \int_{I_{1}} \int_{B_{1}}|\nabla| \nabla u_{R}\right|^{2}\right|^{2} \phi^{4} d x d t \\
\leq C\left\{\left(\int_{I_{1}} \int_{B_{1}}\left|\nabla u_{R}-\bar{\nabla} u_{B_{R}}\right|^{2} d x d t\right)^{1 / 2}+\left(\int_{I_{1}} \int_{B_{1}}\left|\nabla u_{R}-\bar{\nabla} u_{B_{R}}\right|^{4} d x d t\right)^{1 / 2}\right\} \\
\times\left(\left\|\nabla u_{R}\right\|_{L^{2}\left(I_{1} \times B_{1}\right)}+\left\|\nabla u_{R}\right\|_{L^{4}\left(I_{1} \times B_{1}\right)}^{2}\right. \\
\left.+\left\|\nabla u_{R}\right\|_{L^{6}\left(I_{1} \times B_{1}\right)}^{3}+\mid \nabla u_{R}\left\|_{L^{8}\left(I_{1} \times B_{1}\right)}^{4}+\right\| \nabla u_{R} \|_{L^{10}\left(I_{1} \times B_{1}\right)}^{5}\right) .
\end{gathered}
$$

According to the Moser's estimate of Harnack type without involving the mean oscillation:

$$
\left|\nabla u_{R}(0,0)\right| \leq C\left(\int_{I_{1 / 2}} \int_{B_{1 / 2}}\left|\nabla u_{R}(t, x)\right|^{4} d x d t\right)^{1 / 4},
$$

it follows from (4) and the energy inequality (1.3) that

$$
\begin{aligned}
\left|\nabla u_{R}(0,0)\right|^{8} \leq & C E_{0}^{2}\left\|\nabla u_{R}\right\|_{L^{2}\left(I_{1} \times B_{1}\right)}\left(1+\left\|\nabla u_{R}\right\|_{L^{\infty}\left(I_{1} \times B_{1}\right)}+\left\|\nabla u_{R}\right\|_{L^{\infty}\left(I_{1} \times B_{1}\right)}^{2}\right. \\
& \left.+\left\|\nabla u_{R}\right\|_{L^{\infty}\left(I \times B_{1}\right)}^{3}+\left\|\nabla u_{R}\right\|_{L^{\infty}\left(I \times B_{1}\right)}^{4}\right) \\
& \times\left\{\left(\int_{I_{1}} \int_{B_{1}}\left|\nabla u_{R}-\overline{\nabla u}_{B_{R}}\right|^{2} d x d t\right)^{1 / 2}+\left(\int_{I_{1}} \int_{B_{1}}\left|\nabla u_{R}-\overline{\nabla u}_{B_{R}}\right|^{4} d x d t\right)^{1 / 2}\right\} .
\end{aligned}
$$

Here we note that the scaling parameter $R$ is restricted in $\left(0, R_{0}\right)$ in the assumption of theorem. In particular, when $R=M^{-1}$ we have (4.20) so that $\left|\nabla u_{R}(t, x)\right| \leq 2$ over $I_{1} \times B_{1}$,

$$
1=\left|\nabla u_{M^{-1}}(0,0)\right|, \quad\left\|\nabla u_{M^{-1}}\right\|_{L^{\infty}\left(I_{1} \times B_{1}\right)} \leq 2
$$

moreover we have

$$
\left(\int_{I_{1}} \int_{B_{1}}\left|\nabla u_{R}-\overline{\nabla u}_{B_{R}}\right|^{4} d x d t\right)^{1 / 4} \leq 2\left(\int_{I_{1}} \int_{B_{1}}\left|\nabla u_{R}-\overline{\nabla u}_{B_{R}}\right|^{2} d x d t\right)^{1 / 4},
$$

under $R=M^{-1}$. Making scale back in (4) and noting the assumption

$$
1<M \rho_{0},
$$

we see from (4), (4.28), (4.29) and the assumption (4.15) that

$$
\begin{aligned}
1 & =\left(M\left|\nabla u\left(\tilde{t}_{0}, \tilde{x}_{0}\right)\right|\right)^{8} \leq C E_{0}^{2}\left(\frac{1}{R^{2}} \int_{I_{R}\left(t_{0}\right)} \int_{B_{R}\left(x_{0}\right)}\left|\nabla u-\overline{\nabla u}_{B_{R}}\right|^{2} d x d t\right)^{1 / 2} \\
& \leq C \varepsilon_{0}^{\frac{1}{2}} E_{0}^{2}
\end{aligned}
$$

when $M^{-1}=R \in\left(0, R_{0}\right)$. This yields a contradiction when $\varepsilon_{0}$ is sufficiently small. Hence we have the bound $M \rho_{0} \leq 1$. Since $\sigma_{0}$ is the maximizer of $F(r)$ in (4.17),

$$
\left(R_{0}-\frac{R_{0}}{2}\right)^{2} \sup _{P_{R_{0} / 2}}|\nabla u(t, x)| \leq \max _{r \in\left(0, R_{0}\right)}\left(\left(R_{0}-r\right)^{2} \sup _{P_{r}}|\nabla u(t, x)|\right) \leq 1
$$

This shows the boundedness of the derivative of solution

$$
\sup _{P_{\frac{1}{2} R_{0}}}|\nabla u(t, x)| \leq \frac{2^{2}}{R_{0}^{2}}
$$


and hence regularity at $\left(t_{0}, x_{0}\right)$.

\section{CRiterion By MeAN Oscillation}

Proof of Theorem 1.1. We show the proof for the case $M=\mathbb{R}^{2}$. The other cases can be similarly shown. Let $u(t)$ be the global weak solution of (1.2) and $S$ be the set of the points $(t, x) \in[0, \infty) \times \mathbb{R}^{2}$, where the solution is not regular. First we claim that there are at most finitely many singular points over $[0, \infty) \times \mathbb{T}^{2}$. If the weak solution has a singular point for all $t \in\left(0, T_{0}\right]$ for some $T_{0}>0$, then the solution is not continuous in $\mathbb{R}^{2}$ for all $t \in\left(0, T_{0}\right)$. Then since $\|\nabla u(t)\|_{B M O}=\infty$ for every $t \in\left(0, T_{0}\right)$, we have

$$
\int_{0}^{T_{0}}\|\nabla u(t)\|_{B M O}^{2} d t=\infty
$$

which contradicts the assumption (3.2). Therefore the singular points are at most countable. Next if there is an accumulated singular point $\left(t_{0}, x_{0}\right) \in S$, then we may choose a sequence $\left\{\left(t_{n}, x_{n}\right)\right\}_{n} \in S$ and radius $r_{n}>0$ such that the parabolic neighborhoods of the singularity points; $P_{r_{n}}\left(t_{n}, x_{n}\right) \equiv\left(t_{n}-r_{n}^{2}, t_{n}\right) \times B_{r_{n}}\left(x_{n}\right)$ are disjoint each other. Since each vertex of parabolic neighborhood $P_{r_{n}}$ is a singularity of the solution, by Corollary 4.4, we see for some $\varepsilon_{0}>0$ such that

$$
\frac{1}{r_{n}^{2}} \int_{P_{r_{n}}\left(t_{n}, x_{n}\right)}\left|\nabla u(t, x)-\overline{\nabla u}_{r_{n}}\right|^{2} d x d t \geq \varepsilon_{0}
$$

for all $n=1,2, \cdots$. Taking the sum of around the all singularities, we have

$$
\int_{0}^{T_{0}}\|\nabla u(t)\|_{B M O}^{2} d t=\infty
$$

which again contradicts the assumption (3.2) for the weak solution. Hence the singular set $S$ consists of at most finite points in time direction. Analogous argument can be seen to be valid in the space direction for the each singular time $t_{k}$ by using the energy bound:

$$
\sup _{0<t<T}\|\nabla u(t)\|_{2}^{2}<\infty
$$

since $\nabla u \in L^{\infty}\left(0, T ; L^{2}\left(R^{2}\right)\right)$. Therefore the singular points $\left(t_{k}, x_{k}\right) \in S$ are at most finite.

Let $K \in \mathbb{N}$ be the number of those irregular points. We show that under the assumption (3.2), the solution $u$ is regular at those isolated points. Without loss of generality, we consider only the singularities with the smallest time $t_{k}=T_{0}>0$.

Then there exists some small radius $r_{0}>0$ such that any parabolic neighborhood $P_{r_{0}}\left(x_{k}\right) \equiv$ $\left(T_{0}-r_{0}^{2}, T_{0}+r_{0}^{2}\right) \times B_{r_{0}}\left(x_{k}\right)$ centered at $\left(T_{0}, x_{k}\right) \in S$, can be chosen disjointly each other.

Since the weak solution is regular over $\left[0, T_{0}\right)$, from Proposition 3.1, there exists $T>T_{0}$ and a smooth solution $v(t, x)$ of $(1.2)$ over $[0, T)$. All we need to show is that this strong solution indeed coincides the original weak solution $u(t, x)$. We should note that the uniqueness of the weak solution fails in general even on two dimensional manifolds. 
Since $u(t, x)$ and $v(t, x)$ are weak solutions over $\left([0, T) \times \mathbb{R}^{2}\right)$, we can use a test function $u-v$ in the equation obtained from subtracting the ones for $u$ and $v$ to have

$$
\begin{aligned}
\|u(t)-v(t)\|_{2}^{2}+ & 2 \int_{0}^{t}\|\nabla u(t)-\nabla v(t)\|_{2}^{2} d t \\
\leq & 2 \int_{0}^{t} \int_{\mathbb{R}^{2}}|u-v|^{2}|\nabla u|^{2} d x d t+\int_{0}^{t} \int_{\mathbb{R}^{2}}\left(|v|^{2}(|\nabla u|+|\nabla v|)^{2}|u-v|^{2}\right) d x d t \\
& +\int_{0}^{t}\|\nabla u(t)-\nabla v(t)\|_{2}^{2} d t
\end{aligned}
$$

Thus the last term can be cancelled with the term in the left hand side and we let the first and second terms in the right hand side of (5.1) as $I$ and $I I$. We set the irregular part $\cup_{k} P_{r_{0}}\left(x_{k}\right)$ as $\Sigma$. For the first term of the right hand side of (5.1),

$$
I=\iint_{[0, T) \times \mathbb{R}^{2} \backslash \Sigma}|u-v|^{2}|\nabla u|^{2} d x d t+\iint_{\Sigma}|u-v|^{2}|\nabla u|^{2} d x d t .
$$

The second part of the right hand side of (5.2)

$$
\begin{aligned}
\iint_{\Sigma}|u-v|^{2}|\nabla u|^{2} d x d t & \leq \sum_{k=1}^{K} \int_{T_{0}-r_{0}^{2}}^{T_{0}+r_{0}^{2}}\|u(t)-v(t)\|_{\infty}^{2}\|\nabla u(t)\|_{2}^{2} d t \\
& \leq 4\left\|\nabla u_{0}\right\|_{2}^{2} \sum_{k=1}^{K} 2 r_{0}^{2} \leq 8 E_{0} K r_{0}^{2}
\end{aligned}
$$

where we set $E_{0}=\left\|\nabla u_{0}\right\|_{2}^{2}$. While the solutions are regular for the first part,

$$
\begin{aligned}
I & =\iint_{[0, T) \times \mathbb{R}^{2} \backslash \Sigma}|u-v|^{2}|\nabla u|^{2} d x d t+8 E_{0} K r_{0}^{2} \\
& \leq \sup _{\left((0, T) \times \mathbb{R}^{2}\right) \backslash \Sigma}|\nabla u(t)| \int_{0}^{t} \int_{\mathbb{R}^{2}}|u(t)-v(t)|^{2} d x d t+8 E_{0} K r_{0}^{2} .
\end{aligned}
$$

Now we choose $r_{0}$ sufficiently small so that

$$
8 E_{0} K r_{0}^{2} \leq \int_{0}^{T_{0}} \int_{\mathbb{R}^{2}}|u-v|^{2} d x d t
$$

and we fix it. Then we have

$$
r_{0} \leq\left(\frac{1}{8 E_{0} K} \int_{0}^{T_{0}}\|u(t)-v(t)\|_{2}^{2} d t\right)^{1 / 2}
$$

which is independent of $t$. Therefore for all $t \geq T_{0}$,

$$
8 E_{0} K r_{0}^{2} \leq \int_{0}^{t} \int_{\mathbb{R}^{2}}|u-v|^{2} d x d t
$$

From (5.4) and (5.5), we have

$$
I \leq \sup _{\left((0, T) \times \mathbb{R}^{2}\right) \backslash \Sigma}|\nabla u(t)| \int_{0}^{t} \int_{\mathbb{R}^{2}}|u(t)-v(t)|^{2} d x d t+\int_{0}^{t}\|u(t)-v(t)\|_{2}^{2} d t
$$

and

$$
I I \leq\left(\sup _{\left((0, T) \times \mathbb{R}^{2}\right) \backslash \Sigma}|\nabla u(t)|+\|\nabla v\|_{\infty}^{2}\right) \int_{0}^{t} \int_{\mathbb{R}^{2}}|u(t)-v(t)|^{2} d x d t+\int_{0}^{t}\|u(t)-v(t)\|_{2}^{2} d t .
$$


Combining (5.6) and (5.7), we obtain

$$
\|u(t)-v(t)\|_{2}^{2}+\frac{1}{2} \int_{0}^{t}\|\nabla u(t)-\nabla v(t)\|_{2}^{2} d t \leq C_{0} \int_{0}^{t}\|u(t)-v(t)\|_{2}^{2} d t
$$

where $C_{0}=\frac{3}{2} \sup |\nabla u(t)|^{2}+\frac{1}{2}\|\nabla v\|_{\infty}^{2}+\frac{3}{2}$. In particular,

$$
\|u(t)-v(t)\|_{2}^{2} \leq C_{0} \int_{0}^{t}\|u(t)-v(t)\|_{2}^{2} d t .
$$

The Gronwall argument yields $u(t) \equiv v(t)$ over $[0, T)$ for arbitrary $T>0$. Since $v$ is regular this prove theorem.

Acknowledgments. The authors express their thanks to referee for a valuable suggestion on the proof of the regularity criterion. T. Ogawa is partially supported by Grant-in-aid for Scientific Research of JSPS \#15340056 and \#15654023. M.Misawa is partially supported by Grant-in-aid for Scietific Research of JSPS \#15540210.

\section{REFERENCES}

[1] Adams, R.A., Sobolev Spaces, Academic Press, New York, 1975.

[2] Beale, J.T., Kato, T., Majda, A., Remarks on the breakdown of smooth solutions for the 3-D Euler equations, Comm. Math.Phys., 94 (1984), 61-66.

[3] Bergh, J., Löfström, J., Interpolation Spaces, An Introduction , Grundlehren der mathematischen Wissenschaften 223, Springer-Verlag, Belrin-New York- Heidelberg., 1976.

[4] Bertsch, M., Dal Passo, R., van der Hout, R., Nonuniqueness for the heat flow of harmonic maps on the disk. Arch. Ration Mech. Anal. 161 (2002), no. 2, 93-112.

[5] Bertsch, M., Dal Passo, R., Pisante, A., Point singularities and nonuniqueness for the heat flow for harmonic maps. Comm. Partial Differential Equations 28 (2003), no. 5-6, 1135-1160

[6] Brezis, H., Gallouet, T., Nonlinear Schrödinger evolution equations, Nonlinear Anal. T.M. A., 4 (1980), 677-681.

[7] Brezis, H., Nirenberg, L., Degree theory and BMO;Part I: Compact manifolds without boundary Selecta Math. new series, 1 (1995) 197-263.

[8] Brezis, H., Wainger, S., A note on limiting cases of Sobolev embedding and convolution inequalities., Comm. Partial Differential Equations, 5 (1980), 773-789.

[9] Bui Hui Qui, Some aspects of weighted and non-weighted hardy spaces, in "Research of Hardy Spaces and Multi-variable Fourier Analysis by means of Real Analysis (Japanese)" Suuriken Koukyuroku (RIMS Lecture Note, Kyoto Univ.) 383 (1980), 38-56.

[10] Chen, Y-M., Ding, W-Y., Blow-up and global existence for heat flows of harmonic maps. Invent. Math., 99 (1990), 567-578.

[11] Chang, K-C., Ding W-Y., Ye, R., Finite-time blow-up of the heat flow of harmonic maps from surfaces, J. Diff. Geom. 36 (1992), 507-515.

[12] Cheng, X., Estimates of the singular set of the evolution problem for harmonic map, J. Diff.Geom. 34 (1991), $169-174$.

[13] Chen, Y-M., Struwe, M., Existence and partial regularity results for the heat flow for harmonic maps, Math. Z., 201 (1989), 83-103.

[14] Coron, J-M., Ghidaglia, J-M., Explision en temps fini pour le flot des applications harmoniques, C.R.Acad. Sci. Paris, 308 (1989), 339-344.

[15] Eells, J., Sampson, J.H., Harmonic mappings of Riemannian manifolds, Amer. J. Math., 86 (1964), 109-160.

[16] Engler, H., An alternative proof of the Brezis-Wainger inequality, Comm. Partial Differential equations., 14 no. 4 (1989), 541-544.

[17] Evans, L.C., Partial regularity for stationary harmonic maps into spheres, Arch. Rat. Mech. Anal., 116 (1991), 101-113.

[18] Feldman, M., Partial regularity for harmonic maps of evolution into spheres, Comm. Partial Diff. Equations, 19 vol 5\&6, (1994), 761-790.

[19] Freire, A., Uniquness for the harmonic map flow in two dimensions, Clac. Var. 3 (1995) 95-105. 
[20] Giga,Y., Solutions for semilinear Parabolic equations in $L^{p}$ and regularity of weak solutions of the NavierStokes system J. Differential Equations, 62 (1986), 186-212.

[21] Gilbarg, D., Trudinger,N., Elliptic Partial Differential Equation of Second Order, 2nd Ed. Springer-Verlag, Berlin, New York 1983.

[22] Hélein, F., Régularité des applications faiblement harmoniques entre une surface et une sphére. C.R.Acad. Sci.Paris Sér. I, 311 (1990), 519-524.

[23] Kozono, H., Ogawa, T., Taniuchi, Y., The critical Sobolev inequalities in Besov spaces and regularity criterion to some semi-linear evolution equations, Math. Z., 242 (2002), 251-278.

[24] Kozono, H., Taniuchi, Y., Limiting case of the Sobolev inequality in BMO with application to the Euler equations, Comm. Math. Phys. 214 (2000), no. 1, 191-200.

[25] Leray, J., Sur le mouvement d'un liquide visqeux emplissant l'espace., Acta Math., 63 (1934), 193-248.

[26] Ohyama, T., Interior regularity of weak solutions of the time-dependent Navier-Stokes equation, Proc. Japan Acad., 36 (1960), 273-277.

[27] Ogawa, T., Sharp Sobolev inequality of logarithmic type and the limiting regularity condition to the harmonic heat flow, SIAM J. Math. Anal., 34 no. 8 (2003), 1317-1329.

[28] Ogawa, T., Taniuchi, Y., On blow-up criteria of smooth solutions to the 3-D Euler equations in a bounded domain. J. Differential Equations, 190 (2003), 39-63.

[29] Ogawa, T.,Taniuchi, Y., Critial Sobolev inequality and uniqueness problem to the Navier-Stokes equations., Tohoku Math. J., 56 no. 1 (2004) 65-77.

[30] Prodi,G., Un teorema di unicità per le equazioni di Navier-Stokes, Ann. Mat. Pure. Appl., 48 (1959), $173-182$.

[31] Qing, J., On singularity of the heat flow for harmonic maps from surfaces into spheres, Comm. Anal. Geom. 3 no. 2 (1995), 297-315.

[32] Sacks, J., Uhlenbeck, K., The existence of minimal immersion of 2-spheres, Ann. Math., 113 (1981), 1-24.

[33] Schoen, R., Analytic aspects of the harmonic map problem, Publ. M.S.R.I., 2 (1984) 321-358.

[34] Schoen, R., Uhlenbeck, K., A regularity theorem for harmonic maps, J. Diff. Geom. 17 (1982), 307-335.

[35] Serrin,J., On the interior regularity of weak solutions of the Navier-Stokes equations, Arch.Rat.Mech. Anal., 9 (1962), 187-195.

[36] Stein, E.M., Singular integrals and differentiability properties of functions , Princeton University Press, Princton. 1970.

[37] Struwe, M., On the evolution of harmonic mappings of Riemaninan surfaces, Comment. Math. Helvetici, 60 (1985), 558-581.

[38] Struwe, M., On the evolution of harmonic maps in higher dimension, J. Diff. Geom. 28 (1988), 485-502.

[39] Struwe, M., Geometric evolution problems, in "Nonlinear Partial Differential

Equations in Differential Geometry" R.Haardt and M. Wolf eds. pp257-333 IAS/Park City Math. Ser. Vol 2, Ameri. Math. Soc., 1996.

[40] Topping, P., Reverse bubbling and nonuniqueness in the harmonic map flow, Int. Math. Res. Not. (2002), no. 10, 505-520.

[41] Topping, P., Winding behaviour of finite-time singularities of the harmonic map heat flow. Math. Z. (2004), no. $247,279-302$.

[42] Triebel, H., Theory of Function Spaces. Monographs in Mathematics, Birkhäuser-verlag, Basel, 1983. dynamics 